\title{
Sucrose-derived catalytic biodiesel synthesis from low cost palm fatty acid distillate
}

\begin{abstract}
The current homogeneous acid catalyst for biodiesel product however, would lead to formation of many undesirable by-products that make the post treatment of the biodiesel to be difficult and costly. Thus, sucrose-derived solid acid catalyst was developed in the present study which aims to improve the esterification process and reduce the generation of waste. The physicochemical properties of the synthesized catalysts were studied by various techniques such as, BET surface area, X-ray diffraction (XRD), temperature programmed desorption of NH3 (TPD-NH3), scanning electron microscopy (SEM). Response surface methodology (RSM) with central composite design (CCD) is used to determine the optimum parameters for the catalytic reaction. The experimental results showed that the catalyst exhibited good catalytic activity in the transesterification of PFAD, providing maximum biodiesel yield of $94 \%$ at optimum parameters. The better catalytic activity of the aforementioned catalyst in the biodiesel reaction could be attributed to the presence of optimal number of catalytically active acid site density on its surface.
\end{abstract}

